\title{
Estimation of the temperature spatial variability in confined spaces based on thermal imaging
}

\author{
Grzegorz Augustyn ${ }^{1}$, Jakub Jurasz ${ }^{1,}{ }^{*}$, Krzysztof Jurczyk ${ }^{1}$, Tomasz Korbiel $^{2}$, Jerzy Mikulik ${ }^{1}$, Marcin \\ Pawlik $^{1}$, and Rafat Rumin ${ }^{1}$ \\ ${ }^{1}$ AGH University, Faculty of Management, Department of Engineering Management, ul. \\ Mickiewicza 30, 30-059 Kraków, Poland \\ ${ }^{2}$ AGH University, Faculty of Mechanical Engineering and Robotics, Department of Mechanics and \\ Vibroacoustics, ul. Mickiewicza 30, 30-059 Kraków, Poland
}

\begin{abstract}
In developed countries the salaries of office workers are several times higher than the total cost of maintaining and operating the building. Therefore even a small improvement in human work productivity and performance as a result of enhancing the quality of their work environment may lead to a meaningful economic benefits. The air temperature is the most commonly used indicator in assessing the indoor environment quality. What is more, it is well known that thermal comfort has the biggest impact on employees performance and their ability to work efficiently. In majority of office buildings, indoor temperature is managed by heating, ventilation and air conditioning (HVAC) appliances. However the way how they are currently managed and controlled leads to the nonhomogeneous distribution of temperature in certain space. An approach to determining the spatial variability of temperature in confined spaces was introduced based on thermal imaging temperature measurements. The conducted research and obtained results enabled positive verification of the method and creation of surface plot illustrating the temperature variability.
\end{abstract}

\section{Introduction}

The human perception of comfort can be interpreted as a reaction to the observed/noticeable conditions present in given environment. This state results from a multitude of factors such as thermal comfort, air quality, lighting, sounds (noise). More and more often the state of comfort is being represented by a matrix of indices which combine specified parameters describing given surrounding and its conditions. Both, sounds and noise are quantities which can be measured directly and described by a single parameter. Whereas, assessing the quality of climate forces us to consider and combine several factors and parameters.

Accordingly to the norm [1] the thermal comfort can be defined as a state of mind which express satisfaction from the thermal environment. This state is a consequence of an equilibrium between heat generated by human body as a result of metabolism and its loses of heat to the surrounding environment [2]. Tenants who are occupying given building are performing various activities which are associated with varying levels of performed physical activity - which directly translates into generating some quantity of heat. To operate in

*Corresponding author: jjurasz@zarz.agh.edu.pl 
comfortable state, tenants choose their clothing based on performed occupation, age or gender. Those factors may have various impact on the perception of the observed climate in given room. In consequence, it is an arduous task to ensure that all users/tenants will have optimal thermal conditions in their working space. However, appropriately designed heating, ventilating and air-conditioning systems may be a solution which will lead to a situation in which a majority of tenants will feel comfortable [3].

Thermal comfort is influenced by a multitude of factors which in general can be divided into two major categories: environmental ones (air temperature, wind speed, air humidity, surface radiation temperature and the asymmetry of a vertical temperature distribution); personal/individual ones like metabolic rate, acclimatization or clothing insulation. So far a significant amount of papers which has been reviewed $[4,5]$ has been dedicated to the idea of measuring thermal comfort by placing sensors or minimizing the energy consumption. However, they seemed to avoid the problem of an uneven distribution of temperature in various states of given space (heating, cooling etc.) and its impact on the heating, ventilation and air-conditioning systems (HVAC).

This study is a part of a larger project which aim is to establish the certain number and locations of temperature measuring sensors in order to maximize the efficiency of the HVAC system and minimize the energy consumption. Therefore, the aim of the research presented in this study was to assess the validity of thermal imaging in measuring the temperature distribution in confined spaces.

\section{Thermal imaging as a measuring method}

To assess the thermal comfort in given confined space one should use a dedicated tool in form of Microclimate Measure (for example MM-01) [6]. Accordingly to the PN-EN 15251 regulations it is admissible to measure the temperature only when measurements are longlasting and the temperature of radiation can be estimated [7]. It is possible to measure temperature by means of wireless sensors connected to the computer or/and by measuring charts (black matte sheet of paper) which temperature is estimated based on thermal imaging. We have assumed that the radiation coefficient of the considered sheet of paper is equal to $0.9[8,9]$.

\subsection{Thermal imaging}

Temperature can be measured based on thermal imaging, which is a convenient and reasonable solution in research concerning buildings and their interior [10]. For the purpose of this study we have used thermal imaging Flir i7 which measuring range expands from -20 to $+250^{\circ} \mathrm{C}$ and measurements accuracy is $\pm 0.1^{\circ} \mathrm{C}$ and spatial resolution is $140 \mathrm{x} 140$ pixels. The parameters of this comply with PN-ISO 7726:2001 and ASHRAE 55:1992. The potential problem may be the measuring accuracy but it is important to underline that the minimal temperature difference which can be distinguished by a human being is estimated to be $\pm 0.5^{\circ} \mathrm{C}$. According to the [7] and the purpose of our study we set up the measuring range at $0-40^{\circ} \mathrm{C}$ and the measuring accuracy at $\pm 0.4^{\circ} \mathrm{C}$. Measuring black cards have been placed in the system 12/10 (rows/columns) and the distance between cards was evenlyplaced. The total number of measuring points (cards) was 120 .

\subsection{Air flow temperature impact on measurements}

Prior to the measurements in the test room, two independent tests - hot and cold air flow were run in order to confirm the possibilities of using thermal imaging for the purposes of 
thermal comfort calculation. The procedure is based on measurement of matte black sheet of paper. The tests were performed at a height with range from 1 to 2 meters in three different points in the room: 1 - on black matte sample sheet of paper, 2 - on the cooling/heating source, 3 - on the adjacent surface. The objective of these tests was to verify the possibility of performing measurements in changing surroundings conditions (flow of cold or hot air) as shown on Fig. 1. For further tests a procedure based on matt black sheet of paper was used. Which is sensitive to the temperature changes in the measured environment.

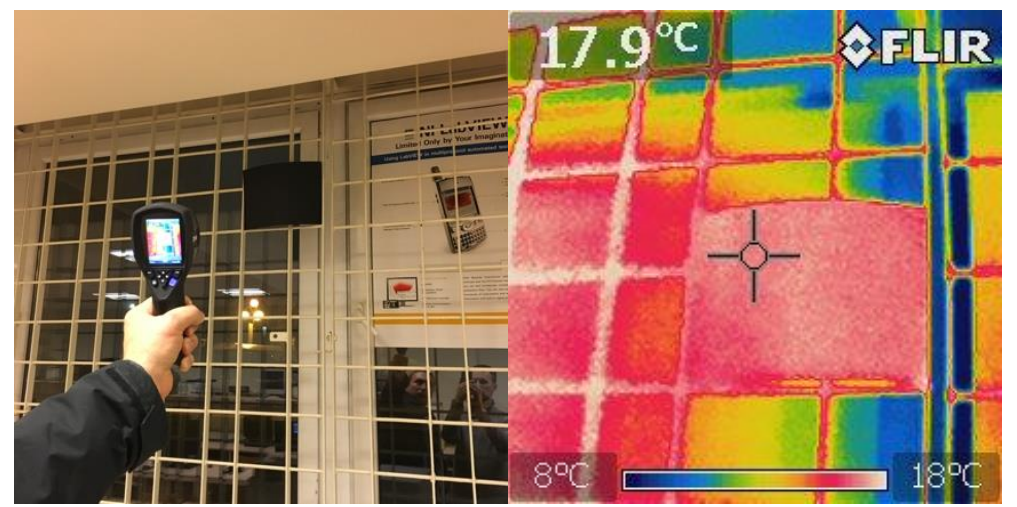

Fig. 1. Temperature measurements near cold source (window).

\subsection{Measuring procedure impact on readouts}

In order to verify the accuracy of results obtained by measuring of the temperature using thermal imaging and check if they are dependent on the measuring angle, distance or influence of hot and cold sources in the imaging field the following experiments have been proposed. Each of them has been repeated five times by two independent operators and realized for a black matte sheet of paper. The aim of this procedure was to check the impact of different measurement positions (angle, distance, etc.) on the results accuracy. An example of performed measurements is presented on the Fig. 2, where Table 1 contains the obtained results based on the below 15 experiments:

1. lens was perpendicular to matte black sheet of paper (referred below to as "it") and they were 0.5 meter apart;

2. lens was perpendicular to it and they were 2.0 meter apart;

3. lens was at $45^{\circ}$ angle to it and they were 0.5 meter apart, irradiation was perpendicular to the camera lens;

4. lens was at $45^{\circ}$ angle to it and they were 0.5 meter apart, irradiation was parallel to the camera lens;

5. lens was at $45^{\circ}$ angle to it and they were 0.5 meter apart;

6. lens perpendicular to it and they were 0.25 meter apart, heat source (radiator) is not visible in thermal imaging;

7. lens perpendicular to it and they were 0.5 meter apart, heat source (radiator) covers $50 \%$ of the taken image;

8. lens perpendicular to it and they were 2.0 meters apart, heat source (radiator) covers is a background of the taken photo;

9. lens perpendicular to it and they were 0.25 meter apart, heat (cold) source (window) is not visible in thermal imaging;

10. lens perpendicular to it and they were 0.5 meter apart, heat (cold) source (window) covers $50 \%$ of the taken image; 
11. lens perpendicular to it and they were 2.0 meters apart, heat (cold) source (window) covers is a background of the taken photo;

12. lens perpendicular to it and they were 2.0 meters apart, heat and cold sources) cover one side of the photo;

13. lens perpendicular to it and they were 0.25 meter apart, heat and cold sources are not visible in thermal imaging;

14. lens perpendicular to it and they were 0.5 meter apart, heat and cold sources are visible in thermal imaging, black matte sheet of paper covers $50 \%$ of image;

15. lens perpendicular to it and they were 0.5 meter apart. There is the same distance from cold and heat source, and both are visible on the photo.

Table 1. Results of the control of measurements accuracy test.

\begin{tabular}{|c|c|c|c|c|c|c|c|c|c|c|c|c|c|c|c|c|}
\hline \multirow{2}{*}{\multicolumn{2}{|c|}{$\begin{array}{l}\text { Operator/ } \\
\text { Measurement } \\
\text { serie }\end{array}$}} & \multicolumn{15}{|c|}{ Experiments } \\
\hline & & 1 & 2 & 3 & 4 & 5 & 6 & 7 & 8 & 9 & 10 & 11 & 12 & 13 & 14 & 15 \\
\hline \multirow{7}{*}{$\mathbf{A}$} & 1 & 21.8 & 22.1 & 22.0 & 22.2 & 22.3 & 27.1 & 26.8 & 27.2 & 22.5 & 23.5 & 23.8 & 26.8 & 25.4 & 25.0 & 25,1 \\
\hline & 2 & 21.9 & 22.1 & 22.0 & 22.0 & 22.3 & 26.9 & 26.7 & 27.3 & 22.7 & 23.4 & 23.7 & 26.8 & 25.3 & 25.0 & 25,1 \\
\hline & 3 & 21.8 & 22.2 & 21.9 & 21.9 & 22.3 & 27.0 & 26.8 & 27.4 & 22.5 & 23.4 & 23.8 & 26.9 & 25.2 & 24.9 & 25,3 \\
\hline & 4 & 21.6 & 22.1 & 22.0 & 22.0 & 22.3 & 27.1 & 26.8 & 27.2 & 22.4 & 23.3 & 23.7 & 26.8 & 25.2 & 24.9 & 25,2 \\
\hline & 5 & 21.7 & 22.1 & 22.0 & 22.0 & 22.3 & 26.6 & 27.0 & 27.2 & 22.4 & 23.4 & 24.0 & 26.7 & 25.3 & 25.0 & 25,3 \\
\hline & Mean & 21.7 & 22.1 & 21.9 & 22.0 & 22.3 & 26.9 & 26.8 & 27.2 & 22.5 & 23.4 & 23.8 & 26.8 & 25.2 & 24.9 & 25,2 \\
\hline & Range & 0.30 & 0.10 & 0.10 & 0.30 & 0.00 & 0.50 & 0.30 & 0.20 & 0.30 & 0.20 & 0.30 & 0.20 & 0.20 & 0.10 & 0,20 \\
\hline \multirow{7}{*}{ B } & 1 & 21.8 & 21.7 & 22.1 & 22.1 & 22.2 & 26.9 & 27.2 & 27.4 & 23.1 & 23.2 & 23.8 & 26.8 & 25.3 & 25.1 & 25,1 \\
\hline & 2 & 21.8 & 21.6 & 21.9 & 21.9 & 22.3 & 26.9 & 27.1 & 27.4 & 23.0 & 23.2 & 23.9 & 26.6 & 25.5 & 25.0 & 25,1 \\
\hline & 3 & 21.9 & 21.7 & 22.0 & 22.0 & 22.5 & 27.0 & 27.2 & 27.4 & 23.0 & 23.3 & 23.9 & 26.7 & 25.3 & 25.2 & 25,0 \\
\hline & 4 & 21.9 & 21.9 & 22.1 & 22.1 & 22.3 & 27.0 & 26.7 & 27.2 & 23.1 & 23.4 & 23.9 & 26.8 & 25.4 & 25.2 & 25,0 \\
\hline & 5 & 21.6 & 21.6 & 22.0 & 22.0 & 22.5 & 27.2 & 27.1 & 27.1 & 23.1 & 23.2 & 23.8 & 26.8 & 25.4 & 25.3 & 25,1 \\
\hline & Mean & 21.8 & 21.7 & 22.0 & 22.0 & 22.3 & 27.0 & 27.0 & 27.3 & 23.0 & 23.2 & 23.8 & 26.7 & 25.3 & 25.1 & 25,0 \\
\hline & Range & 0.30 & 0.30 & 0.20 & 0.20 & 0.30 & 0.30 & 0.50 & 0.30 & 0.10 & 0.20 & 0.10 & 0.20 & 0.20 & 0.30 & 0,10 \\
\hline \multicolumn{2}{|c|}{$\begin{array}{l}\text { Mean } \\
\left(\mathbf{X}_{\text {avg }}\right)\end{array}$} & 21,7 & 21.9 & 22.0 & 22.0 & 22.3 & 26.9 & 26.9 & 27.2 & 22.7 & 23.3 & 23.8 & 26.7 & 25.3 & 25.0 & 25.1 \\
\hline
\end{tabular}

In case of the operator A the mean measured temperature in case of all 15 experiments was equal to $24.2^{\circ} \mathrm{C}$ and their range was $0.22^{\circ} \mathrm{C}$. In case of the operator $\mathrm{B}$ the results were respectively $24.25^{\circ} \mathrm{C}$ and $0.24^{\circ} \mathrm{C}$. For the sake of thermal comfort measurements the obtained results are acceptable because a human being is not capable of distinguishing absolute temperature changes smaller than $0.5^{\circ} \mathrm{C}$. 


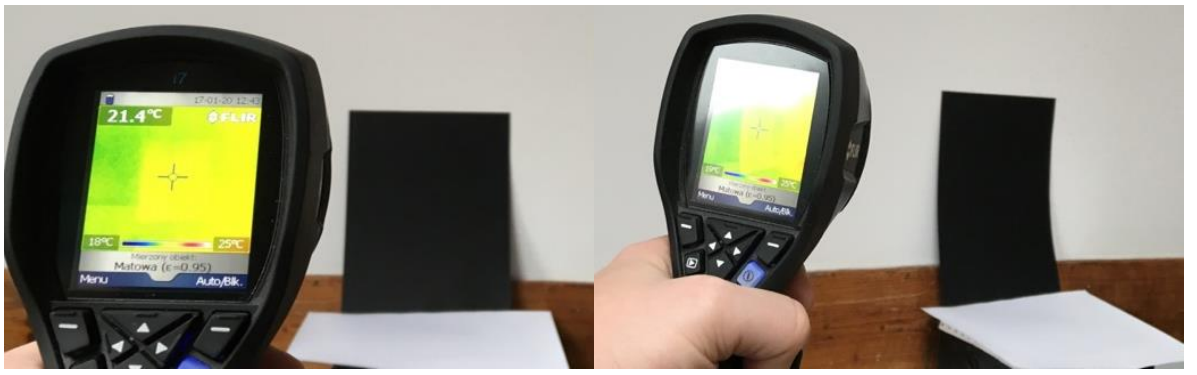

Fig. 2. Experiment no. 1 (left) and 4 (right), thermal imaging position and matte black sheet of paper.

The second test was performed in order to estimate the temperature differences observed on a single measuring black matte sheet of paper, depending on which one out of five points was the lens aimed. Fig. 3 depict the distribution of considered points whereas Table 2 summarizes the results.

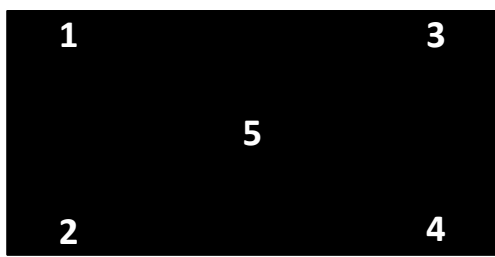

Fig. 3. Location of measuring point during the second part of the experiment.

Table 2. Results of the control of measurements accuracy test - point's location.

\begin{tabular}{|c|c|c|c|c|c|c|}
\hline \multirow{2}{*}{$\begin{array}{c}\text { Operator/ } \\
\text { Measurement serie }\end{array}$} & \multicolumn{4}{|c|}{ Measurement points on the black matte sheet of paper } \\
\cline { 2 - 7 } & 1 & 21.9 & 21.9 & 22.0 & 22.2 & 22.3 \\
\cline { 2 - 7 } & 2 & 21.9 & 21.9 & 22.1 & 22.0 & 22.0 \\
\cline { 2 - 7 } & 3 & 21.7 & 21.9 & 21.8 & 22.0 & 21.9 \\
\cline { 2 - 7 } & Mean & 21.83 & 21.90 & 21.97 & 22.07 & 22.07 \\
\cline { 2 - 7 } & Range & 0.20 & 0.00 & 0.30 & 0.20 & 0.40 \\
\hline \multirow{4}{*}{ A } & 1 & 22.4 & 22.5 & 22.2 & 22.6 & 22.7 \\
\cline { 2 - 7 } & 2 & 22.3 & 22.3 & 22.4 & 22.6 & 22.7 \\
\cline { 2 - 7 } & 3 & 22.4 & 22.2 & 22.5 & 22.2 & 22.3 \\
\cline { 2 - 7 } & Mean & 22.37 & 22.33 & 22.37 & 22.47 & 22.57 \\
\cline { 2 - 7 } & Range & 0.10 & 0.30 & 0.30 & 0.40 & 0.40 \\
\hline
\end{tabular}

The performed tests revealed that in case of the operator $\mathrm{A}$ the mean measured temperature was equal to $21.96^{\circ} \mathrm{C}$ and the set of performed measurements had range of $0.22^{\circ} \mathrm{C}$. The second operator had slightly higher mean and respectively larger range which was $0.3^{\circ} \mathrm{C}$. The results of the conducted tests suggested that the black matte sheet of paper is characterized by a uniform distribution of temperature and this does not impact the measurements accuracy and in consequence they might be used for estimating the temperature. 


\section{Materials - area of study}

All measurements were performed in a lecture hall being a part of the Faculty of Management at the AGH University in Kraków. During measurement the room was not occupied by students and therefore it was possible to simulate the impact of various thermal conditions on the temperature distribution (severe draughts during winter, overheating etc.). The room is controlled by the Building Managements System (BMS). BMS controls a set of convection radiators with electric valves and fan coil unit connected to the building air handling unit installation. In the considered lecturing hall all inlets were located along the northern wall of the room, while outlets are located along the southern one. During measurements the ventilation was operating at its $20 \%$ of capacity (about $200 \mathrm{~m}^{3} / \mathrm{h}$ ) whereas the volume of the lecturing hall is close to $300 \mathrm{~m}^{3}$. The lecturing hall is presented on the Fig.4 in panoramic image.

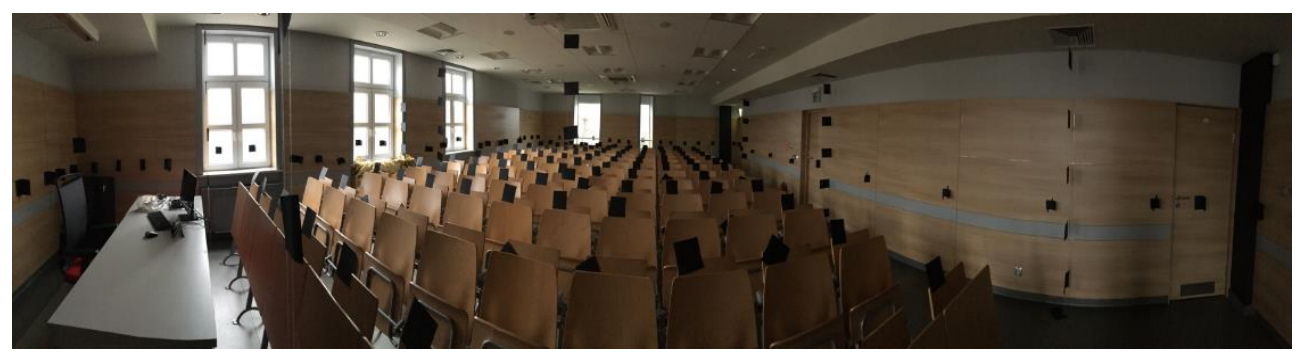

Fig. 4. Lecturing hall where measurements by means of thermal imaging and matte black sheet of paper were performed.

\subsection{Measuring scenarios}

Measurements were taken in an unoccupied lecturing hall (at a stable/fixed state). Over this period the parameters of the HVAC system were as follow: the intake and exhaust of air was $200 \mathrm{~m}^{3} / \mathrm{h}$ which equals to $20 \%$ of the ventilation capacity; the temperature of the supplied air was $20^{\circ} \mathrm{C}$; the radiators was set up in the BMS system to achieve the desired temperature $22^{\circ} \mathrm{C}$.

\section{Results and discussion}

Final measurements were taken in the lecturing hall described in the previous section. Temperature was estimated for an evenly distributed grid of reference points which were located at 1.2 meters above the floor (which is the height at which temperature is usually measured and then used in calculating the thermal comfort). Photos presented on Fig. 5 depict the real object (lecturing hall) and corresponding view from thermal imaging below. Please note that black matte sheet of paper has not have the temperature of the object to which they are attached to, but the surrounding air temperature.

Based on the performed measurements we were able to reconstruct the spatial variability of temperature. For purpose of demonstration we present only results obtained for one of the scenarios explained in the section 3.1. In this scenario measuring sheet of papers were attached to the selected points in the lecturing hall based on previously determined grid. Majority of reference points have been attached to backs of the chairs, where some of them were pinned to the wall surface (as shown in the middle photo on Fig. 5). By attaching them to the wall we were able to determine the temperature of the parietal layer not the wall itself. 

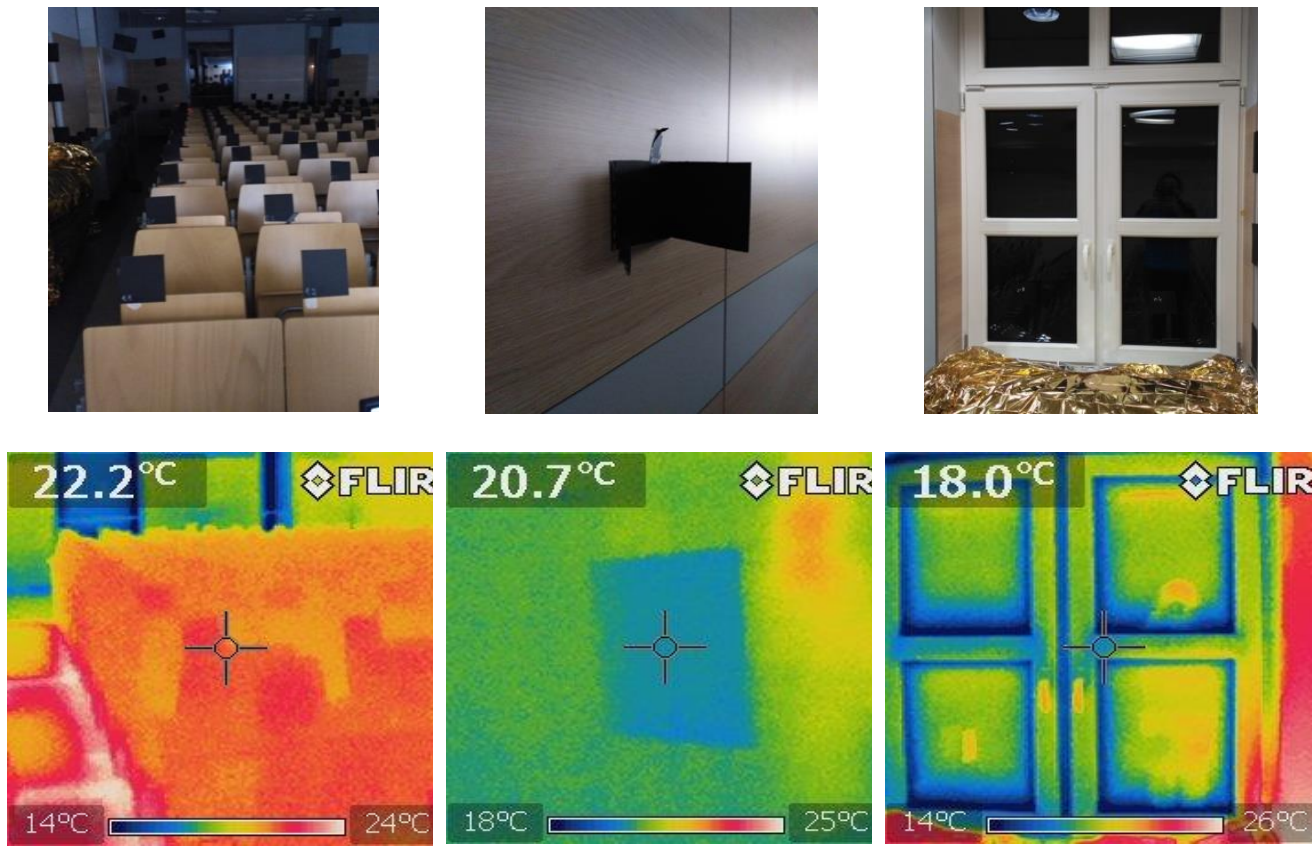

Fig. 5. Demonstrative photo of the lecturing hall with placed measuring sheets of paper in various locations: normal photo and thermal image.

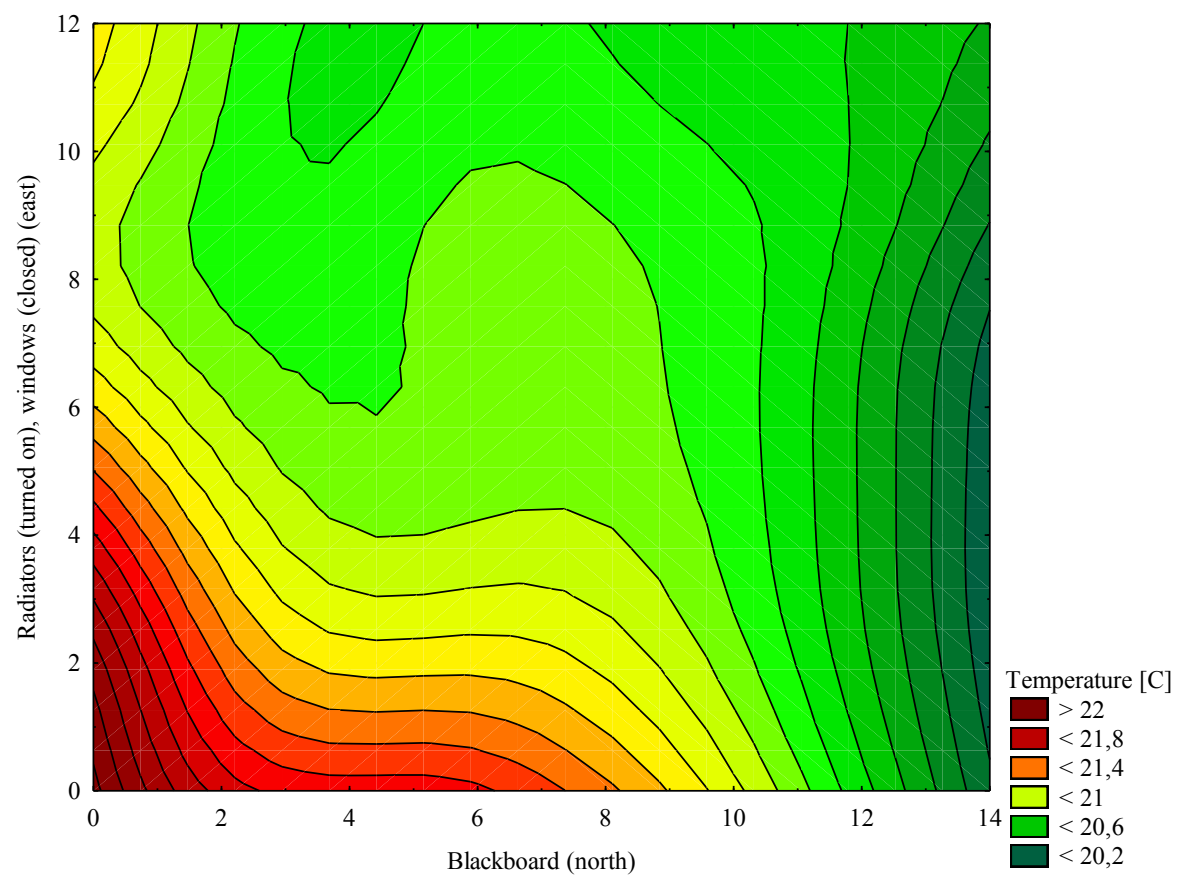

Fig. 6. Temperature distribution in lecturing hall accordingly to the scenario 1.

Additionally, it is important to underline that the sensor which controls the air handling unit was located in the main intake channel of the whole building and as the consequence its 
operation was independent from the temperature observed in the lecturing hall. Sensor used for controlling radiators was located on the east/south corner. Please note, that the temperature observed in this location (Fig. 6. - which was created based on over 120 measuring point and distance-weighted least squares method available in Statistica software) is significantly lower than in the north/east corner were radiators and server (additional heat source) were located. This situation leads to a non-uniform temperature distribution.

The results show the uneven temperature distribution over the room. As the temperature has the major influence on the thermal comfort parameter we can see that measuring the temperature parameter at one single location and supply the feed from the single sensor is inadequate in terms of proper room temperature representation. Other scenarios will very likely confirm that this uneven temperature distribution will increase its impact on the general thermal comfort in the room. The uneven distribution is caused by heating and cooling devices placement but this also affects the thermal comfort for the space around them. In general the common approach assumes the evenly spaced temperature and does not includes the location of heating and cooling sources.

\section{Conclusions}

Obtained results indicate that the presented method is sufficient for performing measurements in lecturing hall. Created chart (Fig. 6) visualized the distribution of temperature in the considered confined space, which is not uniform. Such differences point to the need of using more sensors to correctly operate and control the Building Management Systems (BMS) and ensure that the thermal comfort will be at sufficient level for all occupants. The next step is the verification of the obtained results by comparing them with various measuring tools like InviNets sensors, mercury-in-steel thermometer. Further works will concentrate on investigating various scenarios of room occupancy and their impact on the temperature distribution. Additionally, all measurements will be performed by means of several different measuring systems.

This work was financed by the NCBiR research and scientific project development no. POIR.01.01-00-2008/15 and research grant no. 11/11.200.322

\section{References}

1. W. Olesen, Bjarne Ashrae J. 21-28 (2004)

2. P.O. Fanger, Thermal Comfort (Arkady, 1974) (in Polish)

3. I. Sudoł-Szopińska, A. Bogdan, Bezpieczeństwo pracy, 5 (2007) (in Polish)

4. E. Halawa, J. Van Hoof, Energy and Build. 51, 101-110 (2012)

5. A.K. Mishra, M. Ramgopal, Build. Environ. 64, 94-106 (2013)

6. P. Kiełbasa, P. Budyn, Inżynieria Rolnicza 11, 109, 105-113 (2008) (in Polish)

7. https://www.omega.com/manuals/manualpdf/M5150.pdf User's manual Flir i 7 retrived $\underline{15.02 .2017}$

8. T.S. Wiśniewski, Energia i budynek, 2, (2007) (in Polish)

9. K. Krystian, Instalreporter 10, 59 (2015) (in Polish)

10. M. Pešek, M. Pavelek, J. Eng. Mech.-ASCE 20, 3/4, 327-335 (2013) 